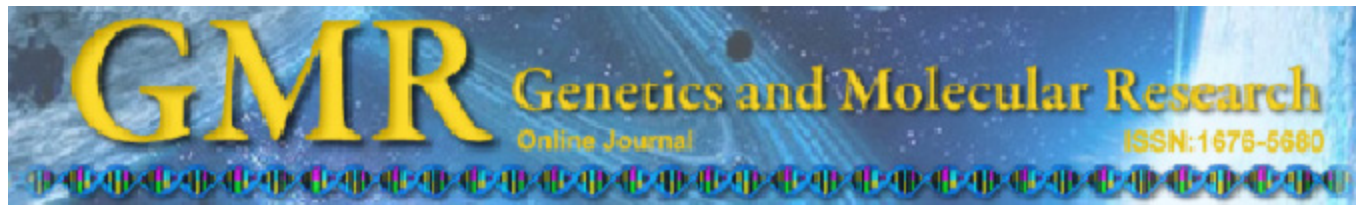

\title{
Effectiveness of olfactory ensheathing cell transplantation for treatment of spinal cord injury
}

\author{
Y.J .Rao ${ }^{1 *}$, W.X. Zhu ${ }^{1 *}$, Z.Q. Du ${ }^{1}$, C.X. Jia ${ }^{1}$, T.X. Du ${ }^{1}$, Q.A. Zhao ${ }^{1}$, \\ X.Y. Cao ${ }^{1}$ and Y.J. Wang ${ }^{2}$ \\ ${ }^{1}$ Orthopedic Hospital of Henan Province, Luoyang, Henan, China \\ ${ }^{2}$ Institute of Spine Disease, Shanghai University of Traditional Chinese \\ Medicine, Shanghai, China \\ *These authors contributed equally to this study. \\ Corresponding author: Y.J. Wang \\ E-mail: yjwcn68@163.com
}

Genet. Mol. Res. 13 (2): 4124-4129 (2014)

Received June 24, 2013

Accepted December 13, 2013

Published May 30, 2014

DOI http://dx.doi.org/10.4238/2014.May.30.7

\begin{abstract}
The aim of this study was to determine the effectiveness and safety of transplantation of olfactory ensheathing cells for functional repair of the spinal cord. An olfactory bulb was obtained from a 4- to 5 -month-old aborted fetus, and it was digested into single olfactory ensheathing cells and then cultured and purified for 1 to 2 weeks. Under general anesthesia, these single-cell suspensions of olfactory ensheathing cells were injected into the corresponding spinal injury site with 0.45-mm-diameter injections. The American Spinal Injury Association (ASIA) Impairment Scale was used to evaluate spinal function. A total of 15 patients (12 men, 3 women; age range, 18-56 years; mean age, 40) were admitted for obsolete spinal injuries. Spinal functions of the 15 patients were observed and followed postoperatively for a period ranging from 2 weeks to 1 month. All the 15 patients exhibited improvements in spinal function, and the improvement tendencies continued. Twelve patients had obvious spinal function improvement, and three had slight improvement according to the ASIA scale, with an
\end{abstract}


obvious difference between preoperation and postoperation measures $(\mathrm{P}$ $<0.05)$. No fevers, infections, functional deteriorations, or deaths were seen. Thus, transplantation of olfactory ensheathing cells promoted spinal and neurofunctional recovery in patients with malignant spinal injuries, and this therapeutic method was safe.

Key words: Olfactory ensheathing cell; Transplantation; Safety; Spinal cord injury

\section{INTRODUCTION}

Spinal cord injuries, both primary mechanical and secondary spinal cord injuries, are severe complications of spinal injuries resulted from the vascular and biochemical reactions occurring after spinal injuries. Nerve cells in the injured segment exhibit necrosis and apoptosis (Emery et al., 1998; Wada et al., 1999). The necrotic and degenerated tissues are removed by phagocytes and replaced by neuroglial cells, leading to the formation of cystic, melanotic, and colloidal lesions at the injured site within 6 weeks after the injury (Wozniewicz et al., 1983; Kinoshita, 1994). Then, the physical separation and neural demyelination interrupt the physiological signal transduction pathway, which is marked clinically by a partial or total loss of sensory, motor, urine, and stool function. Because injured central nerve axons cannot be naturally regenerated (Runyan and Phelps, 2009), functional reconstruction after spinal cord injury has long been a challenging clinical problem.

Basic research in recent years has shown that olfactory ensheathing cell (OEC) transplantation can promote spinal cord functional recovery by changing the pathological process of spinal cord injury. Their wide migration enables post-transplantation OECs to directly occupy the injured site, where they induce axonal extension and control the specific targets of synapses for bridging (Li et al., 1998; Perez-Bouza et al., 1998; Santos-Silva et al., 2007). In addition, OECs directly participate in axonal myelination (Franklin et al., 1996), and they secrete a large number of the factors that are necessary for the growth, development, differentiation, and maturation of different types of neurons and inhibit detrimental factors (Woodhall et al., 2001). OECs provide the necessary conditions for axon or neuron regeneration, facilitating replacement of degenerated neurons or formation of new neurocircuits (Radtke et al., 2009). Thus, they play positive roles in spinal cord regeneration and functional recovery.

OECs, which originate from the mammalian olfactory system, are a type of neuroglial cells that has special functions; they have the dual nature of astroglial cells and Schwann cells (Li et al., 1998; Lu et al., 2001; Keyvan-Fouladi et al., 2003). In recent years, OECs have gradually shown their therapeutic potential in the treatment of spinal cord injuries and thus have become the hot topic of studies on nerve regeneration and repair. Application of OEC transplantation in the treatment of spinal cord injury has yielded some satisfactory results, and it has been considered one of the most promising treatment methods for spinal cord injury (Ramon-Cueto and Avila, 1998; Ramon-Cueto et al., 2000; Hayat et al., 2003). However, many researchers remain skeptical of its curative effects and safety (Dobkin et al., 2006; Fawcett et al., 2007; Steeves et al., 2007).

In order to investigate the effectiveness and safety of OEC transplantation for treatment of spinal cord injuries, we evaluated spinal nerve function in 15 patients with old remote spinal cord injuries who had received OEC transplantation voluntarily. 


\section{SUBJECTS AND METHODS}

\section{Cell culture}

An olfactory bulb was separated from a voluntarily donated human-induced embryo with a 4-5-month gestational age. The bulb was washed, membrane-stripped, cut up, and then washed again under a microscope. The sample obtained was centrifuged, and the supernatant was discarded. Cells were cultured in Dulbecco's Modified Eagle's Medium (DMEM)/F12 medium containing 10\% fetal bovine serum for 2-3 days and then in DMEM OEC-purifying medium for 1-2 weeks. The cells grew well, without any contamination. They were digested, and a monoplast suspension with a concentration of $2 \times 10^{7}$ cells $/ \mathrm{mL}$ was then made.

\section{Subjects}

A total of 15 patients with spinal cord injuries who had received OEC transplantation at the Luoyang Orthopedic-Traumatological Hospital between January and December in 2008 were examined. These patients included 12 men and 3 women, with ages ranging from 18 to 56 years. Their clinical courses ranged from 6 months to 4 years. All the patients had had acute compressive damage caused by their traumatic spinal cord injury. Of the spinal cord injuries, six were located in the cervical segment, two were located in the thoracic segment, and seven were located in the lumbar segment. All the patients had undergone spinal cord decompression and internal fixation after the injury, which was followed by treatment with neurotrophic or nerve growth factors and acupuncture as well as rehabilitation training. All the patients had stable nerve function without compressive lesions at the injured site.

\section{Surgical indications}

Indications for OEC transplantation included a postinjury time of more than 6 months with stable nerve function for more than 3 months, no compressive lesion at the injured spinal cord site, and no transverse anatomic rupture of the spinal cord.

\section{Surgical procedures and cell transplantation}

The surgical procedures were performed under general anesthesia. The patient was placed in a prone position, and a median incision was made. The internal fixers were removed for patients with internal fixers and healed fracture. The upper and lower ends of the injured spinal cord were exposed under the microscope in order to determine the injured region. In order to perform cell transplantation in the injured region, the multi-target point method was adopted. The target points were selected according to the injury (usually at the normal spinal cord around the injured region), and their number was determined depending on the area of the injured region. Approximately $1.0 \times 10^{6}$ cells were injected at each target point (the suspension was $50 \mu \mathrm{L} /$ unit at $2.0 \times 10^{4}$ cells $/ \mu \mathrm{L}$ ), and 2-5 target points were normally selected. Postoperative hemostasis and anti-infection treatment were performed. 


\section{Nerve functional evaluation}

All the patients were followed up for 2-8 weeks after the OEC transplantation. The curative effects were determined by comparing the pre- and post-operative scores, which were based on the American Spinal Injury Association (ASIA) Impairment Scale and injured spinal cord functional rating scale of Xishan Hospital (Chekhonin et al., 2008). The ASIA Impairment Scale score was based on the following: 1) sensory function: Pinprick and touch sensations were tested at each dermatomic key point ( 28 points on each side of the body), and a $0-3$ grade was then assigned $(0=$ no sensation, during which blunt and sharp stimulations could not be differentiated; 1 = sensory disorders; and 2 = normal sensations); the pinprick and touch grades on each side were summed up to get a total sensory grade, which was used to indicate a possible change in sensory function; 2) motor function: Ten key muscles on each side of the body were tested from top to bottom, and a 0-5 grade was then assigned according to strength; the grades on each side were summed up to get a total motor grade, which was used to indicate a possible change in motor function; and 3) the anal sphincter was tested with finger testing. Sensory function was classified into presence and absence, and any sensation in the saddle area indicated an incomplete spinal cord injury. The injured spinal cord functional rating scale consisted of nine categories that were further composed of 16 items. For each item, a four-point system was used, in which a score of 3 meant normal and 0 meant the poorest, with 1 and 2 in between. A total score of 48 (the full score) was considered normal, while scores of 48-35, 34-18, and 17-0 were indicative of slightly, moderately, and severely injured spinal cord, respectively. The scores on this rating scale were used to evaluate whether there was improvement in the patient's urine and stool, muscle tonus, sweating, and skin nutrition.

\section{RESULTS}

\section{Spinal cord functional recovery}

All patients were scored according to the ASIA Impairment Scale and the injured spinal cord functional rating scale of Xishan Hospital 2-8 weeks after OEC transplantation. These scores were then compared with those before the operation. According to the ASIA scores, all patients showed improvements in their spinal cord function after OEC transplantation, with 12 exhibiting significant improvement and three exhibiting slight improvement. The mean ASIA score after OEC transplantation was 16.53 points higher than that before the operation, which was a significant difference $(\mathrm{P}<0.05)$. The scores for the injured spinal cord functional rating scale showed that the mean score after OEC transplantation was higher than that before the operation by 4.42 points, which was a significant difference $(\mathrm{P}<0.05)$. The results are shown in Table 1.

\begin{tabular}{|c|c|c|c|c|}
\hline & Before operation & After operation & Value added & $\mathrm{P}$ \\
\hline ASIA & 160.07 & 176.6 & 16.53 & $\mathrm{P}<0.05$ \\
\hline Spinal cord function evaluation questionnaire & 22.13 & 26.55 & 4.42 & $\mathrm{P}<0.05$ \\
\hline
\end{tabular}




\section{Complications}

None of the patients exhibited postoperative complications, such as long-term fever, spinal cord infection, or functional deterioration. No operation-related deaths occurred.

\section{DISCUSSION}

OECs perform the functions of inducing olfactory axonal extension and controlling the specific in vivo targets of synapses, and these functions are closely correlated with the growth-promoting role of OECs (Wei et al., 2008). OEC membranes express various cellular coherence- and axon growth-related molecules, including FNL1 (fibronectin-like 1), tenascin, laminin, and NCAM (neural cell adhesion molecule). In addition, OECs can secrete many types of nutrition factors, such as nerve growth factor, neurotrophic cytokines, and brain-derived neurotrophic factor; these factors promote the development, differentiation, and survival of neurons. Thus, OECs provide a good internal environment for injured nerve repair, nerve regeneration, and functional recovery (Shyu et al., 2008).

Previous studies have examined effects of OECs transplantation in animal models of local corticospinal lesions, spinal cord transections, and spinal cord contusions and lacerations. OECs transplanted into the injured region of adult rats integrate with host nerve solid tissues to help the regenerated nerve axons penetrate through barriers, such as myelin sheaths and gelatinous tissues; these transplanted OECs then migrate to the specific target region along with axons ( $\mathrm{Li}$ et al., 2008). With regard to neural demyelination, OECs aid myelination of nerves and increase the neural electrophysiology transduction velocity. Thus, OECs play positive roles in nerve repair (neural myelination and nerve regeneration) and spinal cord functional recovery in animal models (Raisman, 2007).

In the present study, the 15 patients with spinal cord injuries who underwent OEC transplantation exhibited different degrees of improvement in spinal cord function. The patients selected in this study had had a primary spinal cord injury for more than half a year and did not show the presence of other compressive factors. Among our patients, the shortest and longest injury time was 6 months and 4 years, respectively; most of the patients had an injury time of over 1.5 years. All the patients had had stable nerve function for more than 3 months. Thus, the possibility that the improvement observed after OEC transplantation was a result of self-recovery could be excluded. Moreover, all the patients had received standard treatments, including surgical decompression and/or neurotrophic drug administration, as well as rehabilitation training, but they did not exhibit positive improvements. Thus, the improvement after OEC transplantation cannot be attributed to spinal cord decompression or rehabilitation training. Interestingly, our study indicated that injured spinal cords, even at late stages of spinal cord contusion and laceration, still have the capacity for nerve repair, regeneration, and functional recovery. This may bring about a gleam of hope for patients at late stages of spinal cord injuries.

In conclusion, OEC transplantation is a safe and feasible surgical procedure for aiding recovery of spinal nerve function in patients with spinal cord injuries.

\section{ACKNOWLEDGMENTS}

Research supported by the Project of Henan Province of China (\#2060502). 


\section{REFERENCES}

Chekhonin VP, Gurina OI, Ykhova OV, Ryabinina AE, et al. (2008). Polyethylene glycol-conjugated immunoliposomes specific for olfactory ensheathing glial cells. Bull. Exp. Biol. Med. 145: 449-451.

Dobkin BH, Curt A and Guest J (2006). Cellular transplants in China: observational study from the largest human experiment in chronic spinal cord injury. Neurorehabil. Neural Repair 20: 5-13.

Emery E, Aldana P, Bunge MB, Puckett W, et al. (1998). Apoptosis after traumatic human spinal cord injury. J. Neurosurg. 89: $911-920$.

Fawcett JW, Curt A, Steeves JD, Coleman WP, et al. (2007). Guidelines for the conduct of clinical trials for spinal cord injury as developed by the ICCP panel: spontaneous recovery after spinal cord injury and statistical power needed for therapeutic clinical trials. Spinal Cord 45: 190-205.

Franklin RJ, Gilson JM, Franceschini IA and Barnett SC (1996). Schwann cell-like myelination following transplantation of an olfactory bulb-ensheathing cell line into areas of demyelination in the adult CNS. Glia 17: 217-224.

Hayat S, Wigley CB and Robbins J (2003). Intracellular calcium handling in rat olfactory ensheathing cells and its role in axonal regeneration. Mol. Cell Neurosci. 22: 259-270.

Keyvan-Fouladi N, Raisman G and Li Y (2003). Functional repair of the corticospinal tract by delayed transplantation of olfactory ensheathing cells in adult rats. J. Neurosci. 23: 9428-9434.

Kinoshita H (1994). Pathology of hyperextension injuries of the cervical spine. Paraplegia 32: 367-374.

Li Y, Field PM and Raisman G (1998). Regeneration of adult rat corticospinal axons induced by transplanted olfactory ensheathing cells. J. Neurosci. 18: 10514-10524.

Li Y, Li D, Khaw PT and Raisman G (2008). Transplanted olfactory ensheathing cells incorporated into the optic nerve head ensheathe retinal ganglion cell axons: possible relevance to glaucoma. Neurosci. Lett. 440: 251-254.

Lu J, Feron F, Ho SM, Mackay-Sim A, et al. (2001). Transplantation of nasal olfactory tissue promotes partial recovery in paraplegic adult rats. Brain Res. 889: 344-357.

Perez-Bouza A, Wigley CB, Nacimiento W, Noth J, et al. (1998). Spontaneous orientation of transplanted olfactory glia influences axonal regeneration. Neuroreport 9: 2971-2975.

Radtke C, Aizer AA, Agulian SK, Lankford KL, et al. (2009). Transplantation of olfactory ensheathing cells enhances peripheral nerve regeneration after microsurgical nerve repair. Brain Res. 1254: 10-17.

Raisman G (2007). Repair of spinal cord injury by transplantation of olfactory ensheathing cells. C. R. Biol. 330: 557-560.

Ramon-Cueto A and Avila J (1998). Olfactory ensheathing glia: properties and function. Brain Res. Bull. 46: 175-187.

Ramon-Cueto A, Cordero MI, Santos-Benito FF and Avila J (2000). Functional recovery of paraplegic rats and motor axon regeneration in their spinal cords by olfactory ensheathing glia. Neuron 25: 425-435.

Runyan SA and Phelps PE (2009). Mouse olfactory ensheathing glia enhance axon outgrowth on a myelin substrate in vitro. Exp. Neurol. 216: 95-104.

Santos-Silva A, Fairless R, Frame MC, Montague P, et al. (2007). FGF/heparin differentially regulates Schwann cell and olfactory ensheathing cell interactions with astrocytes: a role in astrocytosis. $J$. Neurosci. 27: 7154-7167.

Shyu WC, Liu DD, Lin SZ, Li WW, et al. (2008). Implantation of olfactory ensheathing cells promotes neuroplasticity in murine models of stroke. J. Clin. Invest. 118: 2482-2495.

Steeves JD, Lammertse D, Curt A, Fawcett JW, et al. (2007). Guidelines for the conduct of clinical trials for spinal cord injury (SCI) as developed by the ICCP panel: clinical trial outcome measures. Spinal Cord. 45: 206-221.

Wada S, Yone K, Ishidou Y, Nagamine T, et al. (1999). Apoptosis following spinal cord injury in rats and preventative effect of N-methyl-D-aspartate receptor antagonist. J. Neurosurg. 91: 98-104.

Wei Y, Miao X, Xian M, Zhang C, et al. (2008). Effects of transplanting olfactory ensheathing cells on recovery of olfactory epithelium after olfactory nerve transection in rats. Med. Sci. Monit. 14: BR198-BR204.

Woodhall E, West AK and Chuah MI (2001). Cultured olfactory ensheathing cells express nerve growth factor, brainderived neurotrophic factor, glia cell line-derived neurotrophic factor and their receptors. Brain Res. Mol. Brain Res. 88: 203-213.

Wozniewicz B, Filipowicz K, Swiderska SK and Deraka K (1983). Pathophysiological mechanism of traumatic cavitation of the spinal cord. Paraplegia 21: 312-317. 